\title{
The Epidemiology of Hypoparathyroidism in Italy: An 8-Year Register-Based Study
}

\author{
Cristiana Cipriani $^{1}$ (D) Jessica Pepe ${ }^{1} \cdot$ Federica Biamonte $^{1} \cdot$ Rizieri Manai $^{1} \cdot$ \\ Piergianni Biondi $^{1} \cdot$ Luciano Nieddu $^{2} \cdot$ Luisella Cianferotti $^{3} \cdot$ Maria Luisa Brandi $^{3}$. \\ Salvatore Minisola ${ }^{1}$
}

Received: 2 August 2016/ Accepted: 16 December 2016

(C) Springer Science+Business Media New York 2016

\begin{abstract}
Hypoparathyroidism is a rare endocrine disorder, but few studies have focused on the epidemiology and hospital management of the disease and none has been performed in Italy. We investigated the prevalence of different forms of hypoparathyroidism among hospitalized patients in Italy during an 8-year period. This study is designed as a retrospective register-based study. We retrieved data from the "Record of Hospital Discharge" (SDO) of the Italian Health Ministry, from the year 2006 to 2013 and analyzed the codes corresponding to hypoparathyroidism-related diagnoses. The inpatient prevalence of the disease was also calculated after excluding repeated hospitalizations. Overall, 27,692 hospitalization episodes for hypoparathyroidism were identified during the entire period $(72.2 \%$ in women and $27.8 \%$ in men; mean age $49.5 \pm 22.9$ years). The mean length of stay was $7.4 \pm 9.8$ days $(25.9 \%$ of the episodes requiring less than 3 days of stay). The mean hospitalization rate for hypoparathyroidism was 5.9/100,000 inhabitants per year and there was a significant decrease during the period of 2006-2013 $(p<0.0001)$. The mean hospitalization rate for postsurgical hypoparathyroidism was $1.4 / 100,000$ inhabitants per year and the trend showed a significant reduction
\end{abstract}

Cristiana Cipriani

cristiana.cipriani@gmail.com

1 Department of Internal Medicine and Medical Disciplines, "Sapienza" University of Rome, Viale del Policlinico 155, 00161 Rome, Italy

2 Faculty of Economics, UNINT University, Via Cristoforo Colombo 200, 00147 Rome, Italy

3 Metabolic Bone Diseases Unit, Department of Surgery and Translational Medicine, University of Florence, Viale Pieraccini 6, 50139 Florence, Italy during the years $(p<0.0001)$. The mean prevalence of hypoparathyroidism among inpatients was 5.3/100,000 inhabitants per year, and there was a significant decrease over the years $(p<0.0001)$. Hypoparathyroidism, particularly the postsurgical form of the disease, is not an uncommon condition among hospitalized patients in Italy. We observed a tendency to a decrease in the frequency of hospitalization during the period 2006-2013.

Keywords Hypoparathyroidism · Epidemiology · Hospitalization · Italy

\section{Introduction}

Hypoparathyroidism is a rare endocrine disorder characterized by low serum calcium and absent or low serum parathyroid hormone (PTH) levels [1, 2]. Clinical features of the disease include paresthesia, muscle cramps, as well as tetany, seizures, bronchospasm, cardiac arrhythmias, or altered mental status [1]. The acute onset of the severe clinical presentation requiring intravenous calcium administration is particularly common in the postsurgical form of the disease [1,2]. The latter is a not rare complication of surgery for thyroid, parathyroid, and larynx disease and so far represents the most common etiology of hypoparathyroidism [2]. Postsurgical hypoparathyroidism occurs when parathyroid glands are accidentally ablated or damaged during anterior neck surgery and is defined as transient when parathyroid gland function is recovered within weeks or months and chronic when low serum calcium and PTH levels persist 6 months after surgery [1]. Autoimmune hypoparathyroidism can be isolated or present as part of autoimmune polyglandular syndrome (APS) and is characterized by production of anti-parathyroid 
antibodies [1]. Finally, inherited forms of hypoparathyroidism are less common and include genetic defects of the synthesis, secretion or function of PTH, or the development of the parathyroid glands [1]. They can also present in association with genetic defects of other organs and thus identify syndromes with specific genetic characterization [1].

Epidemiology of hypoparathyroidism has been investigated using population-based studies and data from different patients' registries [3-6]. Powers et al. [3] reported a prevalence of 58,793 adults diagnosed with chronic hypoparathyroidism in the period $2007-2008$ in a US health plan database comprising 77 million of patients. Cases of hypoparathyroidism associated with neck surgery were 8901 , of which $25 \%$ were classified as chronic [3]. A prevalence 37/100,000 inhabitants was observed in the Rochester Epidemiology Project in the period 2006-2008, while the Danish Patient Registry data reported a prevalence of 22/100,000 for postsurgical and 2.3/100,000 for non-surgical hypoparathyroidism [4-6].

To date, few data have detailed the prevalence of different forms of hypoparathyroidism, as the majority of the studies mainly focused on the postsurgical form of the disease [3-5]. Moreover, no such study has been performed in Italy. The study was aimed at addressing these issues by defining the prevalence of both postsurgical and primary forms of hypoparathyroidism among hospitalized patients in Italy during an 8-year period. Data from our study also contributed to describing the scenario of the hospital care for hypoparathyroidism and providing information about the burden of the disease for the healthcare system in our country.

\section{Subjects and Methods}

This is a retrospective register-based study on the data from the registry of the Italian Health Ministry, from 2006 to 2013, when the Italian version of the ICD-9 CM classification system was instituted (www.salute.gov). We analyzed the codes corresponding to the following medical diagnoses: "hypoparathyroidism" (252.1), "pseudohypoparathyroidism/ pseudo-pseudohypoparathyroidism" (275.49), "hypocalcemia" associated with neck surgery (275.41), "tetany" associated with neck surgery (781.7), "autoimmune polyglandular syndrome (APS)" (258.1), "DiGeorge syndrome" (279.11), "mitochondrial disorders associated with hypoparathyroidism" [including Kearns-Sayre syndrome and mitochondrial encephalopathy, lactic acidosis, and stroke-like episodes (MELAS) (277.87)]. Cases of hypocalcemia and tetany were retrieved from the database of the Italian Health Ministry only when associated with anterior neck surgery to rule out diagnoses of hypocalcemia or tetany from any other cause by considering the aforementioned ICD codes as the diagnoses of interest in association with the following surgical procedures: "thyroidectomy" (06.39, 06.51), "hemi-thyroidectomy" (06.4, 06.52, 06.6, 06.50, 06.2), "parathyroidectomy" (06.81), "other procedures on the thyroid and parathyroid region" (06.9), section of thyroid isthmus (06.91), "thyroid vessels ligature" (06.92), "thyroid suture" (06.93), "other thyroid procedures" (06.98), "other parathyroid procedures" (06.99), "complete thyroidectomy with laryngectomy" (30.03, 30.04) "laryngectomy + thyroidectomy" $(30.29,30.30,30.40)$. These diagnoses were added in order to avoid underestimation of the results, as many cases of hypoparathyroidism in Italy are diagnosed as "hypocalcemia" or "tetany" and associated with anterior neck surgery, particularly when the complete results of the diagnostic workup are not available. As far as DiGeorge syndrome, APS, and mitochondrial disorders, we included the codes associated with these diagnoses in the estimate only when they were coupled to a diagnosis of hypocalcemia or hypoparathyroidism.

All data were retrieved from the archive of the "Record of Hospital Discharge" ("Scheda di Dimissione Ospedaliera," SDO). Detailed description of the SDO is provided in a previously published paper from our group [7]. Among variables available in the SDO, the following were considered: regional code (region of hospitalization), sex, age, date of hospitalization, date of discharge, days of hospitalization of the patient, and patient's ID.

\section{Statistical Analysis}

The total number of hospitalizations for hypoparathyroidism throughout the 8-year period was evaluated. Patients' mean age is reported as mean \pm SD and as median and interquartile range. The female-to-male (F/M) ratio and days of stay were also assessed.

The percentage of different diagnoses over the total number of diagnoses was calculated. As far as the diagnosis of "hypoparathyroidism" (252.1), the percentage of cases associated with the aforementioned surgical procedures on the anterior neck region was evaluated. The percentage of all diagnoses of postsurgical hypoparathyroidism over the total diagnoses was therefore assessed by including the following: "hypoparathyroidism" associated with anterior neck surgery, "hypocalcemia" and "tetany" associated with anterior neck surgery.

The hospitalization rate for hypoparathyroidism per 100,000 population was calculated using the following formula: (diagnoses/mean annual resident population) $\times 100,000$ inhabitants, by considering the total number of inhabitants in the country for any year, as indicated by the Italian National Statistics Institute database (available online at http://www.istat.it). 
Hospitalization rate per 100,000 inhabitants was calculated for all the hypoparathyroidism-related diagnoses and for postsurgical hypoparathyroidism. The parameter was chosen to providing information on how hypoparathyroidism could impact the healthcare system in our country by defining which proportion of the general population is hospitalized for hypoparathyroidism in the period of interest. The trend of the hospitalization rates over the 8 years was calculated for all the diagnoses and for postsurgical hypoparathyroidism.

The prevalence of all the forms of hypoparathyroidism and postsurgical hypoparathyroidism were calculated by excluding repeated hospitalizations within each year and using the following formula: [diagnoses (after excluding repeated hospitalizations)/mean annual resident population] $\times 100,000$ inhabitants. Patients with repeated hospitalizations for the same diagnosis were identified by specific IDs. Any ID is unique for any patient.

The trend of the prevalence over the 8 years was calculated for all the diagnoses and for postsurgical hypoparathyroidism.

Any of the aforementioned trends was calculated by the nonparametric Pearson's Chi-square test for equality of proportions without continuity correction.

\section{Results}

Table 1 summarizes the number of hospitalization episodes, number of patients (identified after excluding repeated hospitalizations), demographic data, and the length of stay during the 8 years of observation.

The overall number of hospitalization episodes for hypoparathyroidism in the period 2006-2013 was 27,692, the mean number being $3461 /$ year. There was a decrease in the number of hospitalizations during the period of observation, even after excluding repeated hospitalizations (Table 1). Patients' mean age was $49.5 \pm 22.9$ years (Table 1). The female-to-male ratio was 2.6:1. The mean length of stay was $7.4 \pm 9.8$ days $(25.9 \%$ of the episodes requiring $<3$ days of stay). The percentage of diagnoses over the total hospitalization episodes during the 8 years was as follows: "hypoparathyroidism" 59.3\%, "pseudohypoparathyroidism/ pseudo-pseudohypoparathyroidism" $24.4 \%$, "hypocalcemia associated with neck surgery" $14.7 \%$, "tetany associated with neck surgery" $0.6 \%$, "DiGeorge syndrome" $0.7 \%$, "mitochondrial disorders associated with hypoparathyroidism" $0.01 \%$, and "APS" $0.2 \%$ (Fig. 1). Among the diagnoses of "hypoparathyroidism," those associated with neck surgery were $8 \%$ of the total diagnoses (Fig. 1). The percentage of all diagnoses of postsurgical hypoparathyroidism over the total diagnoses was $23.3 \%$. After excluding repeated hospitalizations, the percentage of all patients diagnosed with postsurgical hypoparathyroidism over all patients in the period 2006-2013 was 22.7\%.

Considering all the hypoparathyroidism-related diagnostic codes listed as either the first or other diagnoses, the mean hospitalization rate for hypoparathyroidism was 5.9/ 100,000 inhabitants per year and there was a significant decrease during the period of 2006-2013 (Fig. 2a, $p<0.0001)$. In particular, the mean hospitalization rate for postsurgical hypoparathyroidism was $1.4 / 100,000$ inhabitants per year and the trend showed a significant reduction during the years (Fig. $2 b, p<0.0001$ ).

Figure 3 reports the prevalence of all hospitalized patients with hypoparathyroidism-related diagnoses; the mean prevalence was 5.3/100,000 inhabitants per year, and there was a significant decrease over the years (Fig. 3a, $p<0.0001)$. The mean prevalence of inpatients with postsurgical hypoparathyroidism was $1.3 / 100,000$ inhabitants per year, and we observed a significant reduction over the years (Fig. 3b, $p<0.0001$ ).

Figure 4 shows the mean inpatient prevalence of different forms of hypoparathyroidism per year in Italy calculated after excluding repeated hospitalizations. As far as autoimmune and genetic defects, we observed a mean prevalence of 1.3/100,000 inhabitants per year.

The mean number of hospitalizations was $1.33 \pm 1.27$ per patient in the period 2006-2013. The proportion of patients with more than one hospitalization per year was $8.4 \%$ during the observation.

\section{Discussion}

Our study reports for the first time data on the epidemiology and the hospitalization for hypoparathyroidism in Italy over a long period of time.

Hypoparathyroidism, particularly the postsurgical form of the disease, is not an uncommon condition among hospitalized patients in Italy. Even though the disease is typically mainly managed in the outpatients' setting, the hospitalization rate of 5.9/100,000 inhabitants per year in the period 2006-2013 is not an irrelevant component of the health-related public cost in Italy, being the mean hospitalization rate for any disease 12,285/100,000 inhabitants in the same period (data available at www.salute.gov).

Overall, hypoparathyroidism is mostly diagnosed in women in their middle adulthood. Demographic characteristics of our population are essentially in line with other studies showing an overall higher prevalence of hypoparathyroidism in women (71-88\% depending on studies) in the fifth decade of life $[3,4,6]$. Differences can be otherwise found between other studies and ours in terms of prevalence of the disease [3-6]. According to our data, there was a mean prevalence of hypoparathyroidism among 
Table 1 Total number of hospitalization episodes, patients, demographic data, and length of stay during the observation

\begin{tabular}{|c|c|c|c|c|c|}
\hline Year & $\begin{array}{l}\text { Number of } \\
\text { hospitalization } \\
\text { episodes }\end{array}$ & $\begin{array}{l}\text { Number of } \\
\text { patients }^{\mathrm{a}}\end{array}$ & $\begin{array}{l}\text { Sex }\left(F / M^{b}\right. \\
\text { ratio) }\end{array}$ & $\begin{array}{l}\text { Age (years) } \\
{[\text { mean } \pm \mathrm{SD}} \\
\text { median }(\mathrm{IQR})]\end{array}$ & $\begin{array}{l}\text { Days of stay } \\
{[\text { mean } \pm \mathrm{SD}} \\
\text { median }(\mathrm{IQR})]\end{array}$ \\
\hline 2006 & 3812 & 3413 & $2.6: 1$ & $\begin{array}{l}49.2 \pm 22.7 \\
51(33)\end{array}$ & $\begin{array}{l}7.5 \pm 10.5 \\
5(5)\end{array}$ \\
\hline 2007 & 3976 & 3530 & $2.8: 1$ & $\begin{array}{l}49.8 \pm 22.3 \\
52(32)\end{array}$ & $\begin{array}{l}7.2 \pm 8.9 \\
4(5)\end{array}$ \\
\hline 2008 & 3583 & 3188 & $2.9: 1$ & $\begin{array}{l}50.7 \pm 22.8 \\
53(33)\end{array}$ & $\begin{array}{l}7.7 \pm 10 \\
5(6)\end{array}$ \\
\hline 2009 & 3578 & 3165 & $2.5: 1$ & $\begin{array}{l}49.5 \pm 23 \\
52(33)\end{array}$ & $\begin{array}{l}7.3 \pm 9.7 \\
4(7)\end{array}$ \\
\hline 2010 & 3548 & 3169 & $2.5: 1$ & $\begin{array}{l}50 \pm 23 \\
51(34)\end{array}$ & $\begin{array}{l}7.3 \pm 9.4 \\
4(7)\end{array}$ \\
\hline 2011 & 3279 & 2951 & $2.5: 1$ & $\begin{array}{l}49.3 \pm 22.6 \\
51(32)\end{array}$ & $\begin{array}{l}7.2 \pm 8.4 \\
4(7)\end{array}$ \\
\hline 2012 & 2994 & 2725 & $2.4: 1$ & $\begin{array}{l}49 \pm 23 \\
51(32)\end{array}$ & $\begin{array}{l}7.7 \pm 12.2 \\
4(7)\end{array}$ \\
\hline 2013 & 2922 & 2674 & $2: 5$ & $\begin{array}{l}49.4 \pm 23.4 \\
51.5(33)\end{array}$ & $\begin{array}{l}7.6 \pm 8.9 \\
4(7)\end{array}$ \\
\hline 2006-2013 & 27,692 & 24,815 & $2.6: 1$ & $\begin{array}{l}49.5 \pm 22.9 \\
52(33)\end{array}$ & $\begin{array}{l}7.4 \pm 9.8 \\
4(7)\end{array}$ \\
\hline
\end{tabular}

IQR interquartile range

${ }^{a}$ After excluding repeated hospitalizations

b Female/male

Fig. 1 Percentage of cases of hypoparathyroidism-related diagnoses over the total hospitalization episodes. Backward stripped bar percentage of diagnoses of hypoparathyroidism associated with anterior neck surgery procedures; pseudo-/pseudopseudo-pseudo:

pseudohypoparathyroidism/ pseudo-

pseudohypoparathyroidism; hypocalcemia and tetany: hypocalcemia and tetany associated with anterior neck surgery; APS: autoimmune polyglandular syndrome, MIT: mitochondrial disorders associated with hypoparathyroidism

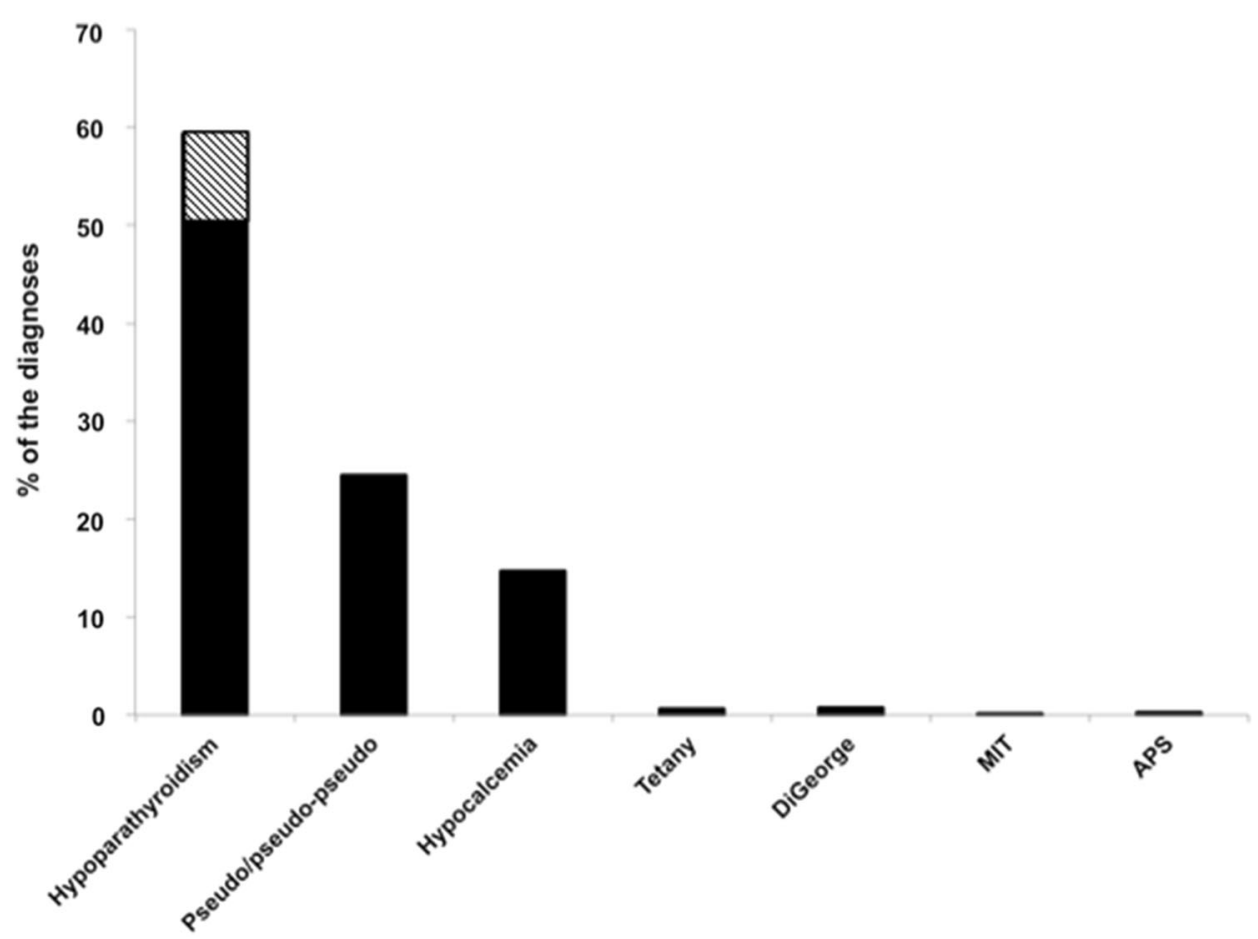



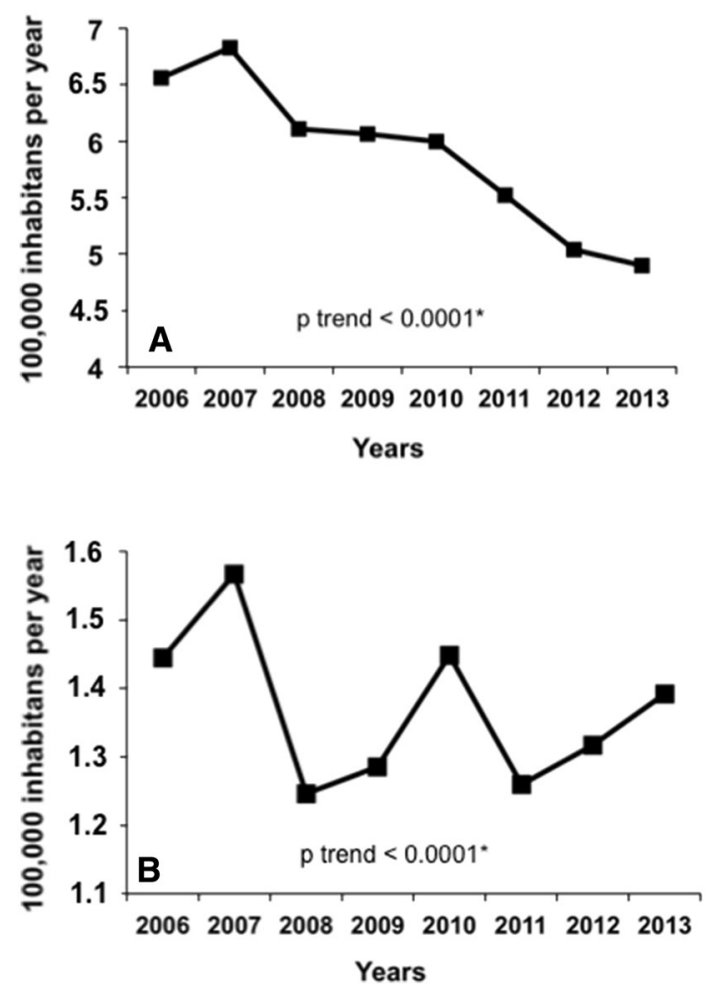

Fig. 2 a Hospitalization rate for hypoparathyroidism/100,000 per year in Italy (considering either the first or other diagnoses). *By test Pearson's Chi-square test for equality of proportions without continuity correction. b Hospitalization rate for postsurgical hypoparathyroidism/100,000 per year in Italy (considering either the first or other diagnoses). *By test Pearson's Chi-square test for equality of proportions without continuity correction

inpatients of 5.3/100,0000/year from 2006 to 2013 in Italy, while other authors reported a higher prevalence [3-6]. These varying results can be ascribed to different population studied, sources of data, and inclusion criteria [3-6]. Indeed, only inpatients were assessed in our study and we did not focus on any specific form of hypoparathyroidism but rather evaluated both primary and secondary conditions. The evaluation of different forms of the disease showed a $23.3 \%$ of cases of hypoparathyroidism associated with an anterior neck surgery procedure. These cases account for all the procedures of the neck region that resulted in impaired parathyroid function and involved a mean of 1.3/100,000 inhabitants per year during the observation. Overall, the prevalence of postsurgical hypoparathyroidism in previous reports varied according to centers of different expertise and experience [1-5, 9-14]. The type and extension of surgery of the neck structures and, consequently, the underlying disease, as well as repeated surgeries, can also affect the rate of postoperative hypoparathyroidism [1, 11, 15]. Powers et al. [3] reported a $7.6 \%$ prevalence of hypoparathyroidism among 117,342 surgeries of the anterior neck region, mostly parathyroidectomy and thyroidectomy. Data from Underbjerg
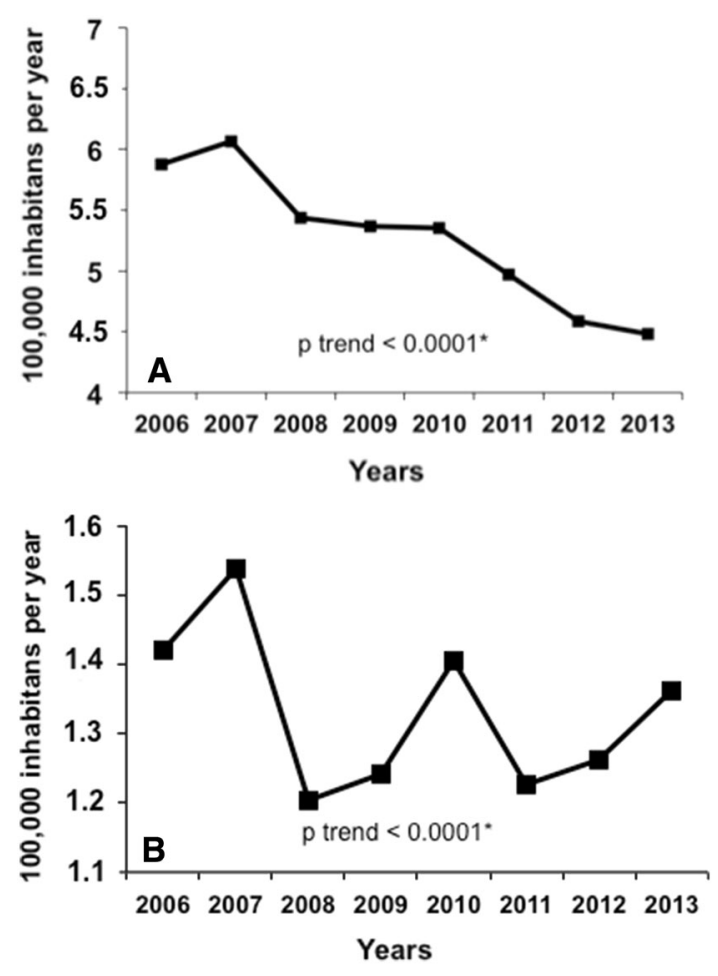

Fig. 3 a Prevalence of hypoparathyroidism/100,000 per year in Italy (excluding repeated hospitalizations) among inpatients. *By test Pearson's Chi-square test for equality of proportions without continuity correction. b Prevalence of postsurgical hypoparathyroidism/ 100,000 per year in Italy (excluding repeated hospitalizations) among inpatients. *By test Pearson's Chi-square test for equality of proportions without continuity correction

et al. [4] showed that the $70 \%$ of patients who developed hypoparathyroidism after surgery had malignant disease. Graves' disease, substernal goiter, as well as the lack of intraoperative PTH monitoring during parathyroid surgery are other risk factors for permanent hypoparathyroidism after surgery $[1,16]$. Our data have been retrieved from the discharge codes of public and private hospitals in the entire Italian area without differentiating between those with high and those with low expertise in endocrine surgery and/or in specific disease of the neck structures. Hence, the results give information on the overall frequency of hypoparathyroidism presenting as an immediate (one or a few days after) complication of neck surgery, as we evaluated all the diagnoses of hypoparathyroidism/hypocalcemia/tetany associated with procedures to the neck region performed during the same hospital admission. As hypoparathyroidism could actually occur several days to weeks after surgery of the neck region [1], it is possible that some of these cases could have been missed. Another limit of the study is represented by the absence of followup data in these patients to assess how many of them developed the chronic form of the disease. However, the accurate inclusion of all the possible forms of the disease 
Fig. 4 Prevalence of different forms of hypoparathyroidism/ 100,000 per year in Italy (excluding repeated hospitalizations) among inpatients

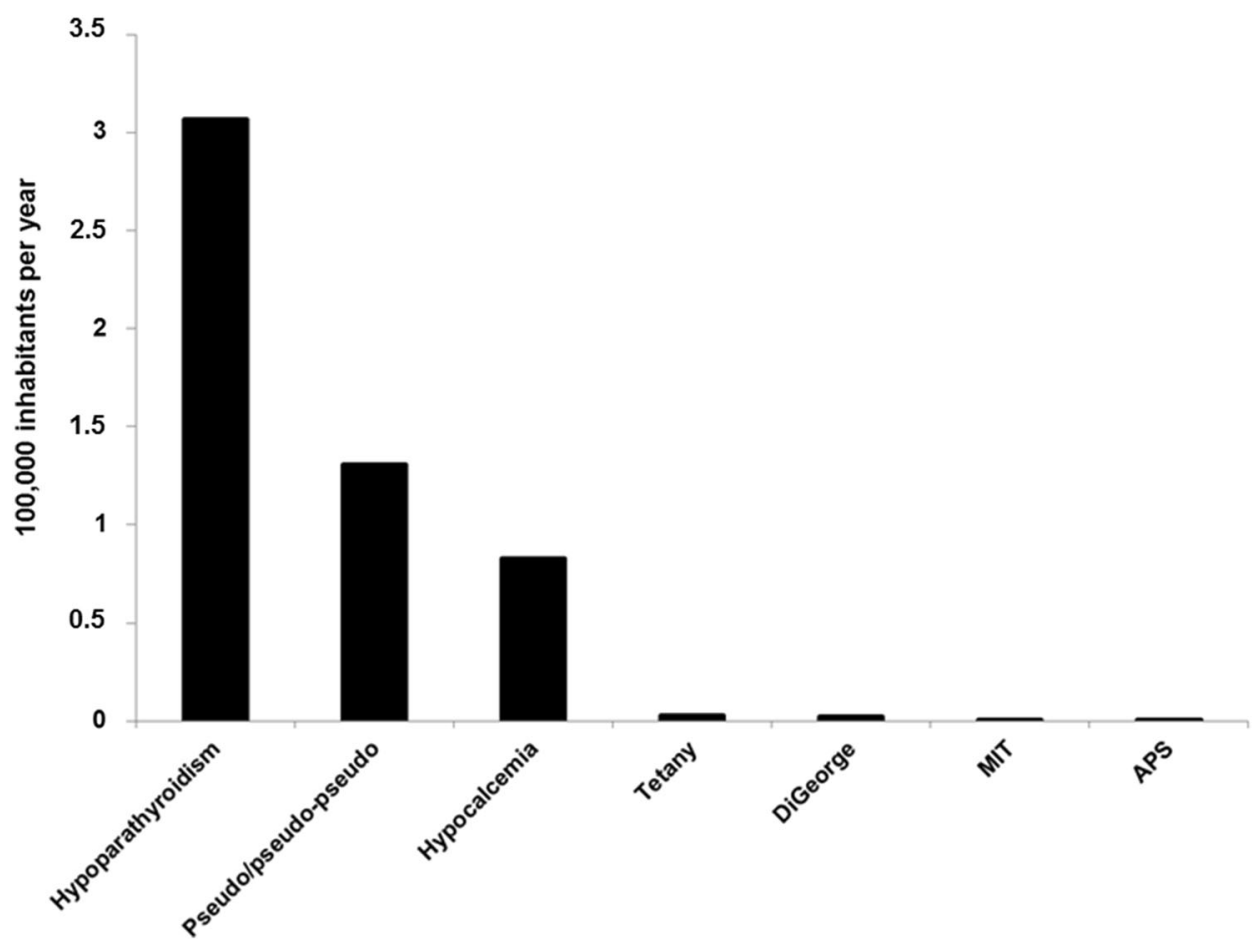

other than postoperative hypoparathyroidism, namely the autoimmune syndromes and genetic defects, allows us to infer that the group of patients with the diagnosis of "hypoparathyroidism" mostly includes patients with chronic postsurgical parathyroid dysfunction. This group represents more than the half of all the diagnoses (59.3\%) and therefore depicts a figure that is essentially in line with previous studies showing a definitely higher proportion of the postsurgical among all other causes of hypoparathyroidism [1-3, 17-19]. Mitchell et al. [17] reported a $66 \%$ rate of postsurgical hypoparathyroidism in a cohort of 120 patients with permanent hypoparathyroidism, while the autoimmune etiology was reported in $7 \%$ of cases, DiGeorge syndrome $3 \%$, and Kearns-Sayre 1\%. Another limitation of our study is that we did not take into account stable and well-controlled patients who may not have required hospitalization and those followed by outpatients' centers and general practitioners. Nevertheless, in our country there is not a dedicated registry for the disease in any region, and regardless of its limitation, the evaluation of the ICD codes is the best way to assess the issue of the epidemiology of the disease on a national basis. Moreover, the $25.9 \%$ of hospitalization episodes with $<3$ days of stay represents patients admitted for hypoparathyroidism, particularly among specialized centers. We can therefore assume that this relevant figure of patients included in our analysis captured those patients admitted for short hospitalizations and managed in a way that is very close to the outpatients' setting.
Additionally, our study provided for the first time data on the inpatient prevalence of those more uncommon causes of hypoparathyroidism in Italy. Epidemiological data on non-surgical hypoparathyroidism come from Underbjerg et al. [5] who reviewed the national hospital registry and individual patient's charts in Denmark between 1977 and 2012. They identified 180 patients with non-surgical hypoparathyroidism and reported an overall prevalence of $2.3 / 100,000$ that is higher to what we observed [5]. Interestingly, pseudohypoparathyroidism/ pseudo-pseudohypoparathyroidism were the most frequent among the non-surgical forms in our study $(1.3 / 100,000$ inhabitants/year). The diagnosis of pseudo-pseudohypoparathyroidism refers to a group of disorders characterized by end-organ resistance to PTH, hypocalcemia, hyperphosphatemia, and high serum PTH, while patients with pseudo-pseudo-pseudohypoparathyroidism have phenotypic appearance of pseudohypoparathyroidism, but are biochemically normal [1, 20]. The analysis of the Danish National Patient Registry identified a total of 60 cases of pseudohypoparathyroidism, equal to a prevalence of $1.1 /$ 100,000 inhabitants [21]. Data from Norway reported similar results [19].

Data on hospitalization also deserve emphasis. We found a mean hospitalization rate for hypoparathyroidism of 5.9/100,000 inhabitants per year in the period 2006-2013 that represents a relevant figure considering the same data in PHPT patients in the period 2006-2011 (12.9/ 100,000) [7]. Hence, hospitalization rate for 
hypoparathyroidism in Italy is almost the half the rate for PHPT, one of the most common endocrine disorders [7, 8]. Different points should be taken into account in explaining why many patients with hypoparathyroidism, a disease usually managed in the outpatient setting, need hospitalization. First, it is possible that the poor metabolic control of the disease lead patients to refer to the hospital and to be admitted. Symptomatic episodes of hypocalcemia and hypercalcemia are indeed common in these patients and are usually associated with inappropriate calcium and vitamin D dosage and/or poor compliance to treatment [17, 22]. This could be the case for patients with repeated hospitalization, who represented a relevant figure $(8.4 \%)$ of these patients in our country. The high prevalence of complications of hypoparathyroidism could also underlie repeated hospitalizations $[6,17,23]$. Many studies reported an increase risk of neuropsychiatric diseases, infections, kidney calcifications, and renal failure [2, 17, 23]. In particular, Underbjerg et al. [23] reported an increased risk of hospitalization due to depression, bipolar disorders, and infections in hypoparathyroid patients. Recurrent infections causing hospitalizations have been also described [23]. Finally, a considerable proportion of hypoparathyroid patients were admitted for surgery at the neck structures; these cases caused an overall 1.3/100,000 hospitalization rate in the period of observation.

The reduction in hospitalization rate, as well as inpatient prevalence of hypoparathyroidism during the years could be associated with a better management of the disease out of the hospital with consequent reduction in acute episodes of hypocalcemia. Also, it is possible that the restriction of public outlays allocated to the healthcare system has led to an overall reduction in hospitalizations or rather a higher need for hospitalizations for other diseases and therefore a decrease in the proportion of inpatients with hypoparathyroidism. Finally, the reduction in cases of hypoparathyroidism after neck surgery over the years could instead be explained by an improvement in surgery techniques and a greater awareness of this possible complication among physicians.

In conclusion, we reported a 5.9/100,000 inhabitants/ year hospitalization rate and 5.3/100,000 inhabitants/year inpatient prevalence of hypoparathyroidism in the period 2006-2013 in Italy. The postsurgical form of the disease represents the most common one. The tendency toward the reduction in hospitalization for hypoparathyroidism of any cause could be attributed to economic issues, but probably also to an improvement in the management of the disease and a reduction in the frequency of complication of surgery. Different factors influencing the epidemiology of hypoparathyroidism and the assessment of the prevalence of long-term complications need to be evaluated in future studies.
Acknowledgements We wish to thank Dr. Flavia Carle and Dr Marco Galadini for their contribution in retrieving data from the Italian Health Ministry database.

Author Contributions CC contributed to design of the work, acquisition, analysis and interpretation of data, and to drafting the manuscript. JP contributed to acquisition of data and revising the manuscript. FB contributed to acquisition of data. RM contributed to design of the work. PB contributed to acquisition of data. LN contributed to data analysis. LC contributed to design of the work and interpretation of data. MLB contributed to project ideation, design of the work, interpretation of data, and revising the manuscript. SM contributed to project ideation, design of the work, analysis and interpretation of data, and revising the manuscript. All the authors have approved the final version of the manuscript.

\section{Compliance with Ethical Standards}

Conflict of interest Cristiana Cipriani, Jessica Pepe, Federica Biamonte, Rizieri Manai, Piergianni Biondi, Luciano Nieddu, Luisella Cianferotti, Maria Luisa Brandi and Salvatore Minisola declare that they have no conflict of interest.

Ethical approval The protocol was performed in accordance with the ethical standards laid down in the 1964 Declaration of Helsinki.

\section{References}

1. Bilezikian JP, Khan A, Potts JT Jr., Brandi ML, Clarke BL, Shoback D, Jüppner H, D'Amour P, Fox J, Rejnmark L, Mosekilde L, Rubin MR, Dempster D, Gafni R, Collins MT, Sliney J, Sanders J (2011) Hypoparathyroidism in the adult: epidemiology, diagnosis, pathophysiology, target-organ involvement, treatment, and challenges for future research. J Bone Miner Res 26:2317-2337. doi:10.1002/jbmr.483

2. Clarke BL, Brown EM, Collins MT, Jüppner H, Lakatos $P$, Levine MA, Mannstadt MM, Bilezikian JP, Romanischen AF, Thakker RV (2016) Epidemiology and diagnosis of hypoparathyroidism. J Clin Endocrinol Metab 101:2284-2299. doi: $10.1210 /$ jc. $2015-3908$

3. Powers J, Joy K, Ruscio A, Lagast H (2013) Prevalence and incidence of hypoparathyroidism in the United States using a large claims database. J Bone Miner Res 28:2570-2576. doi:10. 1002/jbmr.2004

4. Underbjerg L, Sikjaer T, Mosekilde L, Rejnmark L (2013) Cardiovascular and renal complications to postsurgical hypoparathyroidism: a Danish nationwide controlled historic follow-up study. J Bone Miner Res 28:2277-2285. doi:10.1002/ jbmr.1979

5. Underbjerg L, Sikjaer T, Mosekilde L, Rejnmark L (2015) The epidemiology of nonsurgical hypoparathyroidism in Denmark: a nationwide case finding study. J Bone Miner Res 30:1738-1744. doi:10.1002/jbmr.2501

6. Clarke BL et al (2011) Co-morbid- medical conditions associated with prevalent hypoparathyroidism: a population-based study. J Bone Miner Res 26:S182 (Abstract SA1070)]

7. Cipriani C, Carnevale V, Biamonte F, Piemonte S, Pepe J, Nieddu L, Bilezikian JP, Minisola S (2012) Hospital care for primary hyperparathyroidism in Italy: a 6-year register-based study. Eur J Endocrinol 171:481-487. doi:10.1530/EJE-14-0493

8. Clarke BL (2013) Epidemiology of primary hyperparathyroidism. J Clin Densitom. 16:8-13. doi:10.1016/j.jocd.2012.11.009

9. Brandi ML, Bilezikian JP, Shoback D, Bouillon R, Clarke B, Thakker RV, Khan A, Potts JT Jr (2016) Management of 
hypoparathyroidism: summary statement and guidelines. J Clin Endocrinol Metab 101:2273-2283. doi:10.1210/jc.2015-3907

10. Page C, Strunski V (2007) Parathyroid risk in total thyroidectomy for bilateral, benign, multinodular goiter: report of 351 surgical cases. J Laryngol Otol 121:237-241

11. Ito Y, Kihara M, Kobayashi K, Miya A, Miyauchi A (2014) Permanent hypoparathyroidism after completion total thyroidectomy as a second surgery: how do we avoid it? Endocr $\mathrm{J}$ 61:403-408

12. Zarnegar R, Brunaud L, Clark OH (2003) Prevention, evaluation, and management of complications following thyroidectomy for thyroid carcinoma. Endocrinol Metab Clin North Am 32:483-502

13. Paek SH, Lee YM, Min SY, Kim SW, Chun KW, Youn YK (2013) Risk factors of hypoparathyroidism following total thyroidectomy for thyroid cancer. World J Surg 37:94-101. doi:10. 1007/s00268-012-1809-4

14. Thomusch O, Machens A, Sekulla C, Ukkat J, Brauckhoff M, Dralle H (2003) The impact of surgical technique on postoperative hypoparathyroidism in bilateral thyroid surgery: a multivariate analysis of 5846 consecutive patients. Surgery 133:180-185

15. Xiang D, Xie L, Li Z, Wang P, Ye M, Zhu M (2016) Endoscopic thyroidectomy along with bilateral central neck dissection (ETBC) increases the risk of transient hypoparathyroidism for patients with thyroid carcinoma. Endocrine 53:747-753

16. Richards ML, Thompson GB, Farley DR, Grant CS (2008) Reoperative parathyroidectomy in 228 patients during the era of minimal-access surgery and intraoperative parathyroid hormone monitoring. Am J Surg 196:937-943. doi:10.1016/j.amjsurg. 2008.07.022
17. Mitchell DM, Regan S, Cooley MR, Lauter KB, Vrla MC, Becker CB, Burnett-Bowie SA, Mannstadt M (2012) Long-term followup of patients with hypoparathyroidism. J Clin Endocrinol Metab 97:4507-4514. doi:10.1210/jc.2012-1808

18. Rubin MR, Dempster DW, Sliney J Jr, Zhou H, Nickolas TL, Stein EM, Dworakowski E, Dellabadia M, Ives R, McMahon DJ, Zhang C, Silverberg SJ, Shane E, Cremers S, Bilezikian JP (2011) PTH(1-84) administration reverses abnormal bone-remodeling dynamics and structure in hypoparathyroidism. J Bone Miner Res 26:2727-2736. doi:10.1002/jbmr.452

19. Astor MC, Løvås K, Debowska A, Eriksen EF, Evang JA, Fossum C, Fougner KJ, Holte SE, Lima K, Moe RB, Myhre AG, Kemp EH, Nedrebø BG, Svartberg J, Husebye ES (2016) Epidemiology and health related quality of life in hypoparathyroidism in Norway. J Clin Endocrinol Metab 101:3045-3053

20. Mantovani G, Spada A, Elli FM (2016) Pseudohypoparathyroidism and Gs $\alpha$-cAMP-linked disorders: current view and open issues. Nat Rev Endocrinol 12:347-356. doi:10.1038/nrendo.2016.52

21. Underbjerg L, Sikjaer T, Mosekilde L, Rejnmark L (2016) Pseudohypoparathyroidism-epidemiology, mortality and risk of complications. Clin Endocrinol (Oxf) 84:904-911. doi:10.1111/ cen. 12948

22. Bilezikian JP, Brandi ML, Cusano NE, Mannstadt M, Rejnmark L, Rizzoli R, Rubin MR, Winer KK, Liberman UA, Potts JT Jr (2016) Management of hypoparathyroidism: present and future. J Clin Endocrinol Metab. doi:10.1210/jc.2015-3910

23. Underbjerg L, Sikjaer T, Mosekilde L, Rejnmark L (2014) Postsurgical hypoparathyroidism-risk of fractures, psychiatric diseases, cancer, cataract, and infections. J Bone Miner Res 29:2504-2510. doi:10.1002/jbmr.2273 